\title{
Antibacterial resistance: is there a way out of the woods?
}

\section{"We are now facing a crisis that threatens to reverse decades of medical progress and will take clear planning, careful guidance and worldwide cooperation to avert."}

Andrea Marra

Rib-X Pharmaceuticals, 300 George Street, New Haven, CT 06511, USA = Tel.: +1 2038483349 - Fax: +1 2036245627 |. amarra@rib-x.com

Most people in the second half of the 20th century-clinicians and scientists included - would have believed that bacterial infections would be eradicated, or at least easily managed, by the start of the new millennium. Certainly the promise made by the discovery of antibiotics in the 1940s was fulfilled, resulting in millions of lives saved and astonishing medical advances that would have been otherwise impossible. However, in the USA there are close to 2 million hospital-acquired infections and nearly 99,000 deaths yearly, costing US\$20-30 billion, with resistant bacteria being the main culprits [1]. Infections due to these organisms result in longer courses of therapy, increased hospital stays and higher mortality rates than those due to susceptible pathogens. These resistant bacteria are also spreading into the community and affecting healthy people, sometimes with dire consequences; as antibiotic resistance increases so too will these numbers, which are already to high. Yet against this backdrop only two new antibiotics were approved in the last 4 years. We are now facing a crisis that threatens to reverse decades of medical progress and will take clear planning, careful guidance and worldwide cooperation to avert [1].

The unfortunate coincidence of a number of factors that individually would have been significant, in combination could negate the benefits afforded by antibiotics over the last 70 years and lead to increasing numbers of infections caused by multidrug- and pan-resistant organisms. In truth, bacteria resistant to antibacterial drugs will emerge as soon as the two are introduced; if the organism's fitness is not compromised by the resistance determinant, it will have a selective advantage when the antibiotic is present. Conversely, bacteria carrying burdensome resistance determinants can often overcome a fitness cost with compensatory mutations. However, although antibiotic resistance has always been simmering, significant investment in antibacterial research has managed to keep it from boiling over through the steady development of new agents. That is no longer the case as more and more large pharmaceutical companies are exiting the field, due to economic as well as regulatory issues unique to antibacterial drug discovery and development. The Infectious Diseases Society of America (IDSA) has taken on this challenge and issued a set of proposals aimed at increasing awareness and reversing the course of rising antibiotic resistance [1]. The collateral effects of reduced antibiotic efficacy may not be obvious, but they are far-reaching, encompassing procedures such as routine surgery, chemotherapy, intensive care unit therapies, mechanical ventilation, HIV patient care, organ transplant, neonatal/premature infant care, care of the elderly, including residents of long-term care facilities, care of immunocompromised patients and invasive surgical measures - all of which would be impossible without the availability of reliable antibiotics. Even with antibiotics, infectious diseases are the second and third leading causes of death worldwide and in the USA, respectively [2]. To not heed the IDSA's call could result in significant increases in mortality due to infections and a concomitant decrease in life span for the populations of even the wealthiest countries.

\section{What causes antibiotic resistance?}

The challenges posed by antibiotic resistance are exemplified by both Gram-positive and Gram-negative bacterial pathogens. The IDSA has focused on the ESKAPE pathogens - Enterococcus faecium, Staphylococcus aureus, Klebsiella pneumoniae, Acinetobacter baumannii, Pseudomonas aeruginosa and Enterobacter spp. - as these cause the majority of hospitalacquired infections and carry a wide range of troublesome resistance determinants [3]. As an example, the Gram-positive organism $S$. aureus was perhaps the first to gain notoriety due to its acquisition of resistance to $\beta$-lactam drugs. Methicillin-resistant $S$. aureus (MRSA) are now the most common $S$. aureus hospital isolates; more recently, mutations leading to resistance

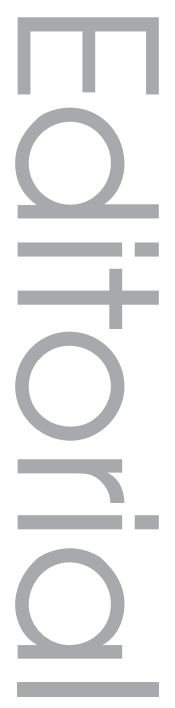

\section{Keywords}

- antimicrobial resistance - ESKAPE pathogens

- multidrug-resistant bacteria

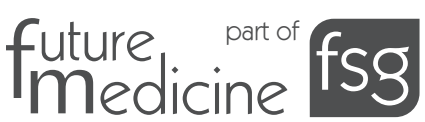


to vancomycin (the workhorse MRSA drug) have been reported. Gram-negative bacteria pose other challenges to drug developers, since, in addition to carrying resistance determinants (carbapenemases, extended-spectrum $\beta$-lactamases or metallo- $\beta$-lactamases) they also have the ability to decrease their susceptibility to antibiotics in a more general fashion by altering membrane permeability and increasing efflux, both of which limit a drug's access to targets inside the cell $[4,5]$.

\footnotetext{
"The recent policy paper published by the IDSA takes a multi-angled look at the issues of rising antibiotic resistance and reduced numbers of new antibiotics..."
}

Resistance can be selected for via mutation, or acquired through genetic transfer and recombination. The most worrisome aspects of the latter are that these mobile genetic elements may contain genes enabling self-replication or chromosomal integration, inter- and intra-species transfer and resistance to multiple antibiotics. It is not unusual to find gene cassettes containing elements conferring resistance to $\beta$-lactams (including extended-spectrum $\beta$-lactamases), aminoglycosides and quinolones; in such cases the only treatment options available are colistin and tigecycline and these are limited by toxicity and low blood levels, respectively [4]. Recent reports of colistin and tigecycline resistance, though isolated, are warnings that time is running out with respect to pan-resistant pathogens.

\section{Why are pharmaceutical companies abandoning this area?}

Since millions of bacterial infections are treated every year, it would seem that the antibiotic market would be a lucrative investment for pharmaceutical companies. Indeed, for several decades many companies were active in this area and reported steady pipelines that led to very potent, life-saving antibiotics. The stream slowed in the 1990s for a number of reasons: first, antibiotic drug discovery is difficult rather than a single target, antibiotics must inhibit a multispecies target, with different active sites, cell permeabilities, efflux potentials and resistance determinants in each organism. This takes time, and discovery groups in large pharmaceutical companies are often held to aggressive timelines to move compounds into the clinic. A second challenge for this therapeutic area is the high dose required in order to achieve blood levels necessary for efficacy, which can lead to difficult-to-manage side effects. Third, pharmaceutical companies saw huge financial successes with 'blockbuster' drugs - compounds that manage health issues but do not cure them and thus must be administered over years or even decades. Antibiotics, on the other hand, eradicate infections and are generally given for a limited course of no more than 1 week. Since the development of any new drug costs close to $\$ 1$ billion, the pursuit of antibiotics offers a significantly lower return on investment. Fourth, antibiotic use leads to antibiotic resistance - no other drug class has that effect - and so a novel, efficacious agent may be held in reserve (further limiting sales and profits) in favor of a cheaper drug. This point is exacerbated by the fact that efforts to reduce healthcare costs will likely further limit use of new antibiotics due to their higher cost over older or generic drugs. Fifth, given that antibiotic resistance generally occurs incrementally, companies typically pursued a strategy of making marginal changes to existing compounds, so as to avert resistance mechanisms while retaining the same favorable safety profile and/or mechanism of antibacterial action. This was a successful strategy for many years; however, recent clinical trial requirements by the US FDA state that new agents must demonstrate superiority over existing agents, effectively torpedoing potential drugs that have equivalent efficacy and may in fact serve a needy patient population now or in the future. The result is that of the 20 pharmaceutical companies involved in antibacterial research in 1990, only two remain active today [101].

\section{How has the regulatory environment affected this?}

Clinical trials cost companies a lot of money. As mentioned, antibiotics' lower return on investment compared with other drugs can mean the difference between a company's continued commitment to or exit from the area. In addition, the FDA's delay in issuing guidance documents outlining appropriate study design and acceptable efficacy outcomes is an ongoing issue, as are perceived inconsistencies in expectations for different companies by the agency [2]. The requirement that studies demonstrate superiority over a currently used agent results in trials that are larger in patient number, costlier and longer in duration than many companies can afford. Clarity around acceptable study design, validated outcome measurements and 
swifter turnaround from the FDA are reasonable expectations and would benefit all parties involved [6].

\section{What does the IDSA recommend?}

The recent policy paper published by the IDSA [1] takes a multi-angled look at the issues of rising antibiotic resistance and reduced numbers of new antibiotics, to propose a comprehensive set of recommendations aimed at reversing this alarming trend. The recommendations include strengthening and/or approving the Generate Antibiotic Incentives Now (GAIN) and Strategies to Address Antimicrobial Resistance (STAAR) Acts; appointing leaders and panels to coordinate efforts to improve infection control and to monitor, collect and report on antimicrobial resistance and priority pathogens across the country; focusing the FDA's efforts on clear communications and guidance; creating an Antimicrobial Innovation and Conservation fee to help offset costs of drug development and appropriate stewardship; generating incentives for companies to continue working in this area; supporting development of rapid diagnostic tests that would allow more appropriate antibiotic use; establishing guidelines for organism-specific, rather than infection site trials; and eliminating use of antibiotics in otherwise healthy livestock.

These recommendations are not meant to be a wish list, but rather a purposeful set of aims that are achievable and whose successes would be readily measureable. The days when a clinician could reach for any number of antibiotics to treat an infection are over; now, more judicious and appropriate antibiotic stewardship is necessary so as not to squander the vast benefits that antibiotics confer. Concerted and disciplined efforts by researchers, legislators, clinicians and patients are required at this critical time.

\section{Financial \& competing interests disclosure}

The author has no relevant affiliations or financial involvement with any organization or entity with a financial interest in or financial conflict with the subject matter or materials discussed in the manuscript. This includes employment, consultancies, honoraria, stock ownership or options, expert testimony, grants or patents received or pending, or royalties.

No writing assistance was utilized in the production of this manuscript.

\section{Bibliography}

1. Infectious Diseases Society of America. Combating antimicrobial resistance: policy recommendations to save lives. Clin. Infect. Dis. 52(Suppl. 5), S397-S428 (2011).

2. Spellberg B, Guidos R, Gilbert D et al. The epidemic of antibiotic-resistant infections: a call to action for the medical community from the Infectious Diseases Society of America. Clin. Infect. Dis. 46, 155-164 (2008).
3. Boucher HW, Talbot GH, Bradley JS et al. Bad bugs, no drugs: no ESKAPE! An update from the Infectious Diseases Society of America. Clin. Infect. Dis. 48, 1-12 (2009).

4. Arias CA, Murray BE. Antibiotic-resistant bugs in the 21st century - a clinical super-challenge. N. Engl. J. Med. 360 (5), 439-443 (2009).

5. Maltezou HC. Metallo- $\beta$-lactamases in Gram-negative bacteria: introducing the era of pan-resistance? Int. J. Antimicrob. Agents 33, 405.E1-405.E7 (2009).
6. Cooper MA, Schlaes D. Fix the antibiotics pipeline. Nature 472, 32 (2011).

\section{Website}

101. Lifesaving antibiotics face doubtful future. Infectious Diseases Society of America Press Release. 7 April 2011 www.idsociety.org/Content.aspx?id=17577 